\title{
Approaching the dynamics of hot nucleons in supernovae
}

\author{
M. Liebendörfer ${ }^{\mathrm{a}}$, U. Pen ${ }^{\mathrm{a}}$ and C. Thompson ${ }^{\mathrm{a}}$ \\ ${ }^{a}$ CITA, University of Toronto, Toronto, Ontario, M5S 3H8, Canada
}

All recent numerical simulations agree that stars in the main sequence mass range of $9-40 \mathrm{M}_{\odot}$ do not produce a prompt hydrodynamic ejection of the outer layers after core collapse and bounce. Rather they suggest that stellar core collapse and supernova explosion are dynamically distinct astrophysical events, separated by an unspectacular accretion phase of at least $\sim 40 \mathrm{~ms}$ duration. As long as the neutrinospheres remain convectively stable, the explosion dynamics is determined by the neutrons, protons, electrons and neutrinos in the layer of impact-heated matter piling up on the protoneutron star. The crucial role of neutrino transport in this regime has been emphasized in many previous investigations. Here, we search for efficient means to address the role of magnetic fields and fluid instabilities in stellar core collapse and the postbounce phase.

\section{Introduction}

The complicated trajectory through core collapse determines the state of the cold nuclear matter deep inside the protoneutron star. At its surface and above, the hot mantle of shock-dissociated nucleons grows by the continued accumulation of infalling matter, heated by the impact at the fairly stationary accretion front at a radius of order 100 $\mathrm{km}$. Simulations with general relativistic neutrino transport in spherical symmetry have explored, in detail, the important role of weak interactions between neutrinos and nuclear matter in stratified layers of curved space-time [1, 2]. Before $50 \mathrm{~ms}$ after bounce, the entropy of the shocked matter is even higher than the entropy that would be achieved by infinitely long exposure to the prevailing neutrino field. After that, the neutrinospheres recede to smaller radii and produce higher luminosities. The accretion front moves to larger radius where the gravitational potential is weaker and the infalling matter has less kinetic energy. Only after that time, do the conditions develop for neutrino heating to become effective. As illustrated in Fig. 1, incoming fluid elements experience an entropy increase toward the equilibrium entropy set by the prevailing neutrino abundances. If the fluid element is compressed during infall, antineutrinos are preferentially absorbed to adjust the electron fraction in response to the increased electron Fermi energies; if it expands, more neutrinos are absorbed. The infalling fluid element crosses the equilibrium entropy at the gain radius. Due to the small reaction time scale in the cooling region, the state of the fluid element approximately follows the local equilibrium entropy and electron fraction thereafter until the neutrinos become trapped behind the energy-dependent neutrinospheres. The caption of Fig. 1 encapsulates the well-known reasons why spherically symmetric supernova models have failed to explain the observed explosions. 

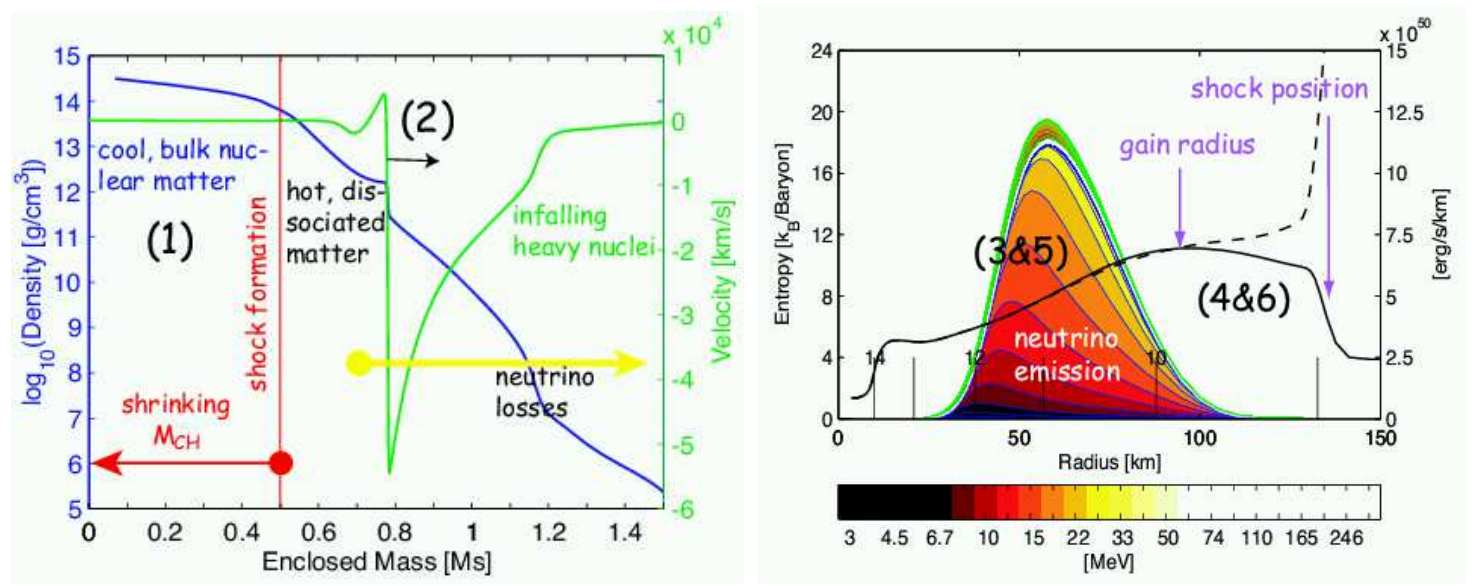

Figure 1. Supernova models have to overcome at least one of six obstacles before they can explode. These are: (1) electron capture during collapse, (2) dissociation of nuclear matter by the shock, (3) neutrino cooling of accreted material, (4) fast accretion velocities, (5) the stability of the neutrinospheres, (6) technical challenges with the neutrino transport. The graph on the left hand side shows a density and velocity profile right after bounce, the graph on the right hand side shows the entropy profile (solid line) at $100 \mathrm{~ms}$ after bounce. The dashed line indicates the equilibrium entropy. A more detailed description of the presentation is given in Ref. [3].

Spherically symmetric simulations ignore fluid instabilities (for a recent discussion of convection in the heating region and protoneutron star see e.g. Refs. [4] and [ 5] respectively). In spite of the agreement that fluid instabilities favor explosions, the latter references suggest that they might develop more slowly than hitherto assumed, leading to failed supernova explosions in two-dimensional simulations as well. The computation time spent on hydrodynamics is likely to be negligible in accurate supernova simulations in one or even two dimensions. Most time is spent on energy-dependent neutrino transport. A systematic improvement of the neutrino transport from one to two dimensions alone required a substantial increase of computation time in yet incomplete implementations [ 4, 6] and three-dimensional neutrino transport has not yet been attempted with a reliable resolution of the neutrino phase space. Following the three-dimensional simulations of [ 7], we try to balance the computation time spent on hydrodynamics and neutrino transport by maximizing the degrees of freedom in the fluid dynamics in combination with approximations in the neutrino transport. The effect of magnetic fields on the dynamics of the nucleons in the hot mantle has not yet been studied in three-dimensional numerical simulations with neutrino transport approximations.

\section{An exploratory three-dimensional simulation with magnetic fields}

A simple and fast three-dimensional magneto-hydrodynamics code [ 8] provides the core of our simulations. It spans a central region of $(600 \mathrm{~km})^{3}$ with an equidistant resolution of $1 \mathrm{~km}$ in Cartesian coordinates. This covers the hot mantle and part of the 
infalling layers. The code has received a new parallelization with MPI for cubic domain decomposition that minimizes the resources occupied on distributed memory machines by a simple and efficient reuse of buffer zones during the directional sweeps. The finite differencing is second order accurate in time and space and handles discontinuities in the conservation equations with a total variation diminishing scheme. Furthermore, a specific choice of the finite differencing for the update of the magnetic field conserves its divergence to machine precision. A velocity decomposition in the spirit of [9] has been implemented such that the entropy equation is solved for a smooth large-scale bulk velocity and the total energy equation for small-scale velocity perturbations. We embed the computational domain of the MHD code in spherically symmetrically infalling outer layers that are evolved by an implicitly finite differenced one-dimensional hydrodynamics code [10].

Our collapse simulations are launched from a $13 \mathrm{M}_{\odot}$ progenitor model [ 11]. The Lattimer-Swesty equation of state [12] is used. We imposed a rotation with angular velocity $\Omega=4 \mathrm{~s}^{-1}$ along the z-axis with a quadratic cutoff at $100 \mathrm{~km}$ radius. Along the same axis, we added a homogeneous magnetic field of $10^{12}$ Gauss. The deleptonization during collapse has been parameterized in a simplistic way: An investigation of the spherically symmetric model N13 [ 2] with Boltzmann neutrino transport reveals that the electron fraction during infall can roughly be approximated as a function of density $\rho$. In our three-dimensional simulation, we update the electron fraction with $Y_{e}(x, y, z)=\min \left[Y_{e}(x, y, z), Y_{e}^{N 13}(\rho(x, y, z))\right]$, where the function $Y_{e}^{N 13}(\rho)$ has been read out of model N13 at the time of core-bounce. The effectiveness of this simple parameterization for this exploratory simulation is demonstrated in Fig. 2.

Due to the rotation, the polar infall velocities are slightly larger than the equatorial infall velocities. When the central density reaches $10^{11} \mathrm{gcm}^{-3}$ the magnetic field lines become visibly distorted. Due to the centrifugal forces, the projection of the velocities onto the plane orthogonal to the initial magnetic field is largest at about $100 \mathrm{~km}$ above and below the gravitational center. These are then the locations where the magnetic field lines condense most rapidly, bending slightly outward around the center. With ongoing collapse, this effect shifts to smaller radii and becomes more pronounced. At bounce, the magnetic field exceeds $10^{15}$ Gauss in these hot spots located $\sim 10 \mathrm{~km}$ above and below the center. The field lines run along double cones aligned with the z-axis, except for the small deviation that circumvents the center. In the early shock expansion until $5 \mathrm{~ms}$ after bounce, the shock front is almost spherically symmetric. Afterward, the simulation becomes unrealistic, because the dynamically important neutrino burst is completely ignored. Behind the expanding accretion front, entropy variations due to variations in the shock strength induce fluid instabilities that entangle the magnetic field lines. Fig. 3 shows an example snapshot at $15 \mathrm{~ms}$ after bounce.

The simulations have been performed on 64 processors of the 528 processor McKenzie cluster at CITA. They required a wall clock time of 150 hours. We demonstrated that three-dimensional magnetohydrodynamics simulations in the supernova context are feasible at a comfortable spatial resolution and that one-dimensional hydrodynamics is likely to find a natural successor in future investigations of stellar core collapse. As the neutrino interactions in the postbounce phase are crucial and very sensitive to the neutrino energies, we plan to continue with a multi-group neutrino light-bulb simulation to study 


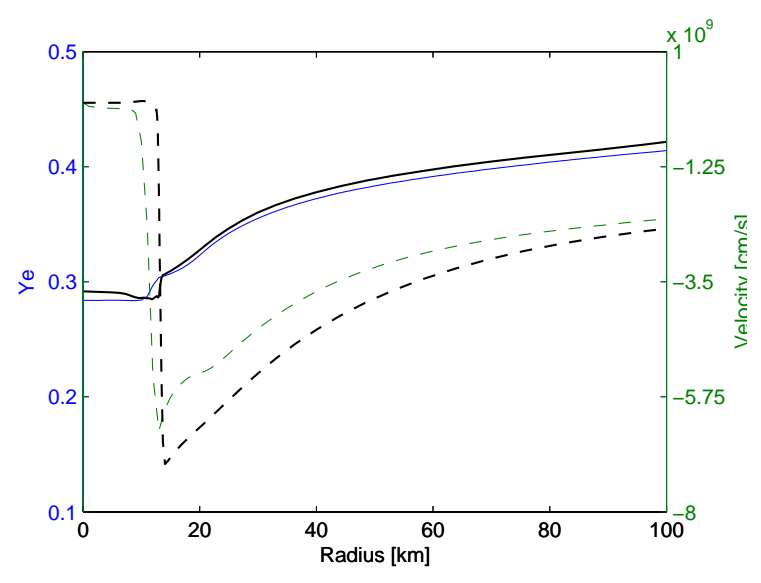

Figure 2. Electron fraction (solid) and velocity (dashed) profiles are compared between a spherically symmetric simulation with accurate neutrino transport (thick lines) and the spherically averaged quantities in the 3D MHD simulation (thin lines) at bounce.

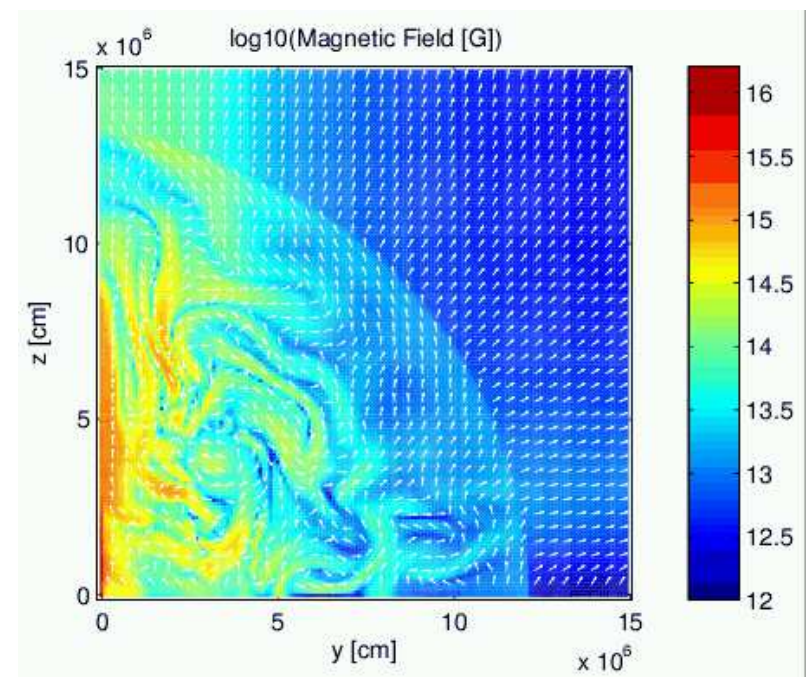

Figure 3. The gray scale in the graph represents the logarithmic amplitude of the entangled magnetic field in the $\mathrm{y}-\mathrm{z}$ plane at $15 \mathrm{~ms}$ after bounce. The arrows indicate the directions of the field lines. No symmetry is imposed in this three-dimensional simulation.

on a more realistic numerical foundation interesting suggested effects of magnetic fields in the hot mantle (see e.g. Refs. [13, 14, 15, 16]).

\section{REFERENCES}

1. S. W. Bruenn, K. R. DeNisco and A. Mezzacappa, ApJ 560 (2001) 326.

2. M. Liebendörfer, M. Rampp, H.-T. Janka and A. Mezzacappa, astro-ph/0310662

3. M. Liebendörfer, Messer, Mezzacappa, et al., ApJS 150 (2004) 263.

4. R. Buras, Rampp, Janka and Kifonidis, Phys. Rev. Lett. 90 (2003) 241101.

5. S. W. Bruenn, E. A. Raley and A. Mezzacappa, astro-ph/0404099.

6. E. Livne, Burrows, Walder, Lichtenstadt and Thompson, ApJ 609 (2004) 277.

7. C. F. Fryer and M. S. Warren, ApJL 574 (2002) 65.

8. U. Pen, P. Arras and S. Wong, ApJS 149 (2003) 447.

9. H. Trac and U. Pen, New. Astron. 9 (2004) 443.

10. M. Liebendörfer, S. K. Rosswog, and F.-K. Thielemann, ApJS 141 (2002) 229.

11. K. Nomoto and M. Hashimoto, Phys. Rep. 163 (1988) 13.

12. J. Lattimer and F. D. Swesty, Nucl. Phys. A535 (1991) 331.

13. C. Thompson, ApJ 534 (2000) 915.

14. S. Akiyama, J. C. Wheeler, D. L. Meier and I. Lichtenstadt, ApJ 584 (2003) 954.

15. T. A. Thompson, E. Quataert, A. Burrows, astro-ph/0403224.

16. K. Kotake, Yamada, Sato, Sumiyoshi, Ono, Suzuki, Phys. Rev. D 69 (2004) 124004. 